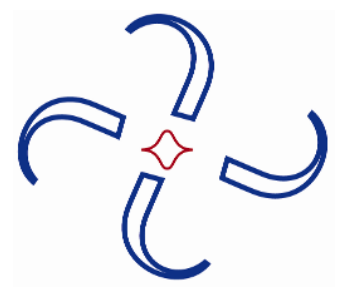

\title{
COLLECTIVE DYNAMICS IN LIPID MEMBRANES: FROM PORE FORMATION TO FLIP-FLOPS
}

\author{
Andrey A. Gurtovenko ${ }^{1,2}$ and Ilpo Vattulainen ${ }^{3,4,5}$ \\ ${ }^{1}$ Institute of Macromolecular Compounds, Russian Academy of Sciences, \\ St. Petersburg, Russia; ${ }^{2}$ Computational Biophysics Laboratory, Institute of \\ Pharmaceutical Innovation, University of Bradford, West Yorkshire, \\ United Kingdom; ${ }^{3}$ Department of Physics, Tampere University of \\ Technology, Tampere, Finland: ${ }^{4}$ Helsinki University of Technology, \\ Helsinki, Finland; ${ }^{5}$ MEMPHYS - Center for Biomembrane Physics, \\ University of Southern Denmark, Odense, Denmark
}

\subsection{INTRODUCTION}

Biological membranes are excellent examples of biologically relevant soft interfaces. They mediate or even govern a large variety of cellular functions [1-3]. Membranes serve as a host for membrane proteins to carry out their functions, and numerous signaling processes are either conducted inside membranes or mediated by them. Additionally, cellular membranes act as a permeability barrier, allowing only desired particles to permeate through the membrane into and out of the cell, besides which membranes are also involved in a variety of large-scale functions such as in maintaining the osmotic pressure and ion density gradients across the plasma membrane.

The biological relevance of membranes is emphasized by the rather recently proposed lipid raft model [4-7], which essentially stresses the importance of understanding the interplay between lipids and proteins: membrane proteins function together with lipids. Consequently, lipid membrane structures, lipid domain coexistence, and especially the role of cholesterol in the structural properties of membranes have been paid a considerable amount of attention recently. Meanwhile, the dynamics of membranes [3,8,9] has received much less attention despite its substantial importance in, e.g., signaling, domain formation, and diffusion of lipids and proteins in the plane of the membrane. 
In this contribution, we discuss the dynamics of lipids in particular. We first consider some of the most relevant dynamic processes where lipids are involved in biological membranes, and then move on to discuss some dynamic processes in detail, in particular those dealing with the formation of pores in membranes [10,11]. It turns out that the nature of dynamic phenomena taking place in membranes is largely collective. They cannot be described as a set of singleparticle (single-lipid) processes that are largely independent from each other. Instead the actual dynamics of lipids involves large assemblies of molecules where the lipids move in unison in a collective manner. Several examples of dynamic phenomena discussed below show that this is a generic feature rather than an exception: dynamic membrane processes such as pore formation and flip-flops illustrate this view and especially highlight the importance of understanding the dynamics of lipids from a collective perspective.

\subsection{DYNAMICS OF LIPIDS IN MEMBRANES}

Membranes, like all biological and soft matter, are governed by weak interactions of the order of thermal energy, $k_{\mathrm{B}} T$. In the same spirit, the dynamics of lipids in membranes is mainly driven by thermal fluctuations. This is truly exciting since it implies that Nature uses random walks in a variety of dynamical processes: the diffusion of individual lipids in a membrane is a random walk, the ordering and formation of membrane domains is partly related to random walks, and also membrane proteins move in a random walk-like manner.

It has to be emphasized that there is no specific timescale characterizing the dynamics of lipids. Instead, the timescales depend on the dynamic process in question as well as on the length scale involved. One of the fastest dynamic processes is the rotational diffusion of individual carbon-hydrogen bonds in $\mathrm{CH}_{2}$ groups in lipid hydrocarbon chains. The timescale of these rotational motions is usually on the order of picoseconds in fluid-like membranes [12,13], though it can be considerably slower in the gel phase below the main transition temperature. The rotational motion of whole lipids around their principal axes of rotation is a slower process and takes place over a scale of nanoseconds $[14,15]$. Meanwhile, the timescale of lipid lateral diffusion depends on the length scale considered. For a given diffusion length $\ell_{\mathrm{D}}=(2 d D t)^{1 / 2}$, where $d$ is the dimensionality, $D$ the diffusion coefficient, and $t$ the timescale of diffusion, it is obvious that the timescale and the range of diffusion given by $\ell_{\mathrm{D}}$ are coupled. For a lipid in a fluid membrane, the diffusion coefficient is typically about $10^{-7} \mathrm{~cm}^{2} / \mathrm{s}$, thus it diffuses over a distance of its own size (about $0.8 \mathrm{~nm}$ ) in about $15 \mathrm{~ns}$. However, as biological membranes are heterogeneous and characterized by membrane domains, the more relevant timescale is the time needed to cross a domain. Assuming a domain to have a size of about $50 \mathrm{~nm}$, a lipid would on average diffuse across it in about $60 \mu \mathrm{s}$. Further, given a cell with a radius of $10 \mu \mathrm{m}$, a lipid would diffuse to the opposing side in about a minute. In strongly ordered lipid rafts the timescales would be larger by a factor of 10 , since in these systems $D \approx 10^{-8} \mathrm{~cm}^{2} / \mathrm{s}[16,17]$. These examples illustrate how the timescales of lateral diffusion increase with the length scale considered $\left(t \propto \ell_{\mathrm{D}}^{2}\right)$. Yet, there is reason to stress that diffusion provides an effective means for transport over cellular scales, since a timescale of the order of seconds is modest compared to times related to the folding of proteins, for instance. Additionally, as far as passive diffusion is concerned, no ATP is wasted: for a cell the motion of lipids comes for free, since the motion of lipids is driven by thermal fluctuations. 
While the lateral diffusion of lipids is characterized by a wide range of timescales ranging from nanoseconds to minutes, there are considerably slower dynamic processes in a cell membrane, characterized by hours and even days. In this contribution, we consider one such phenomenon, the translocation (flip-flop) of lipids from one membrane leaflet to another. While it may sound surprising that atom-scale simulations can shed light on dynamic phenomena that take place on macroscopic timescales, it turns out that simulations can indeed complement experimental studies and provide added value in order to better understand the principles guiding complex dynamic processes in lipid membranes.

\subsection{FLIP-FLOPS ASSOCIATED WITH ASYMMETRIC DISTRIBUTION OF LIPIDS IN MEMBRANES}

One of the dynamical processes that we consider in more detail below is a flip-flop. In general, biological membranes of most animal cells are characterized by an asymmetric distribution of lipid molecules across a membrane $[18,19]$. This asymmetry is vital for numerous cellular functions and plays an important role, e.g., in membrane mechanical stability [20], membrane electrostatics [21-23], and modulation of the activity of membrane proteins [24]. Failure to maintain the asymmetric distribution of lipids can have dramatic consequences. For example, it has been shown that the externalization of anionic phosphatidylserines, which are normally localized in the inner leaflet of plasma membranes, is able to trigger programmed cell death [25].

To maintain the asymmetric transmembrane lipid distribution, the cell needs to be able to translocate lipid molecules from one membrane leaflet to another. One way of doing this is to employ special cellular mechanisms for active transport of lipids across biological membranes using specific membrane proteins - flippases [26,27]. In addition, cells also use passive transport mechanisms that facilitate the migration of lipids across a membrane; such passive translocations may take place with the help of proteins or without them [28].

There is reason to emphasize that the molecular mechanisms associated with passive transmembrane transport of lipids in protein-free membranes are exceptionally poorly understood. A thorough understanding of these mechanisms is one of the key challenges in cell biology [29]. Lipid flip-flops in the absence of flippases are rather difficult to characterize as they take place over molecular scales and often cannot be well resolved within existing experimental methods. On the computational side, the atomistic modeling of lipid flip-flops is usually considered to be prohibitive in terms of accessible timescales: lipid flip-flops in general are very slow processes characterized by an average waiting time ranging from several hours to several days [30,31]. In turn, the state-of-the-art atomic-scale simulations for systems of moderate size (20000-40000 atoms) are still limited to a timescale of about a microsecond.

However, there is strong experimental evidence that lipid translocation across a protein-free membrane is a pore-mediated process. It has been found that the activation energy for radioactive chloride flux across lipid membranes is close to the activation energy of lipid flip-flop [30], implying that the occurrence of pore defects is part of the flip-flop process. It has also been demonstrated that brief electric pulses are able to enhance the transbilayer mobility of phospholipids through electroporation of membranes [32]. Furthermore, recent computer simulations support the idea that pore formation and lipid flip-flop are coupled: when a lipid was dragged by an external force through the hydrophobic membrane interior, the formation 
of a small water pore was observed; the corresponding energies of the formation of the pore and of the lipid transmembrane translocation were found to be the same [33]. Taken together, these findings strongly suggest that a major fraction of lipid flip-flops takes place through water pores in membranes.

Remarkably, it turns out that formation of transient water pores is the rate-limiting step in the process of transmembrane lipid flip-flop [33,34]: once a pore has been formed, the subsequent lipid translocation occurs on timescales accessible through state-of-the-art computer simulations. Below, we demonstrate how this takes place. In particular, we use atomistic computer simulation data to discuss the complexity of lipid flip-flops, which turns out to be a highly collective process involving not only lipids but also their interplay with ions and water. Essentially, we will find that flip-flops take place through water pores generated by a locally strong transmembrane potential, which in turn is induced by an ion concentration gradient across a membrane. Without a doubt, this process is very appealing due to its collective character, which is likely a rule rather than an exception in biological systems in general.

\subsection{FORMATION OF TRANSIENT WATER PORES IN LIPID MEMBRANES}

We now discuss some practical applications of computer simulations for studying the dynamics of lipids in membranes. In particular, we highlight recent findings that have shed light on the formation of transient pores in membranes, and the consequent lipid flip-flops taking place through the pores.

On the computational side, various methods have been used to induce transient hydrophilic defects in lipid bilayers. In particular, pore formation has been observed under conditions where a bilayer has been subjected to an external electric field (electroporation) [35-37] or mechanical stress (surface tension) [35,38,39]. Here we consider a different scenario where pore formation is induced by a transmembrane ionic charge imbalance without any external forces $[10,11]$. The imbalance of ions across a membrane is an inherent feature in living cells. It is believed that the ion concentration differences determine the resting membrane potential in cells [40]. The average value of the potential is of the order of $100 \mathrm{mV}$. However, due to fluctuations its instantaneous value can deviate considerably from the average.

To characterize pore formation, let us consider recent results $[10,11]$ based on atomic-scale molecular dynamics (MD) simulations on lipid bilayers comprised of zwitterionic dimyristoylphosphatidylcholine (DMPC) lipids. The temperature is $323 \mathrm{~K}$, which is well above the main transition temperature of a DMPC bilayer (297 K) [41], meaning that the simulated DMPC bilayers are in the fluid phase.

There is reason to mention one practical issue that is relevant here. Because of periodic boundary conditions, it is not feasible to incorporate a transmembrane ionic charge imbalance into a conventional bilayer simulation (a single lipid bilayer in a box of water): salt ions on one side of the bilayer would diffuse to the other side within a nanosecond time span, thereby discharging the ionic imbalance. The way forward is to use a double-bilayer setup, that is, two lipid bilayers in the same simulation box $[42,43]$ (see Fig. 5.1). This setup provides a means to control electrolyte compositions in the two water phases on the opposite sides of the membrane. 


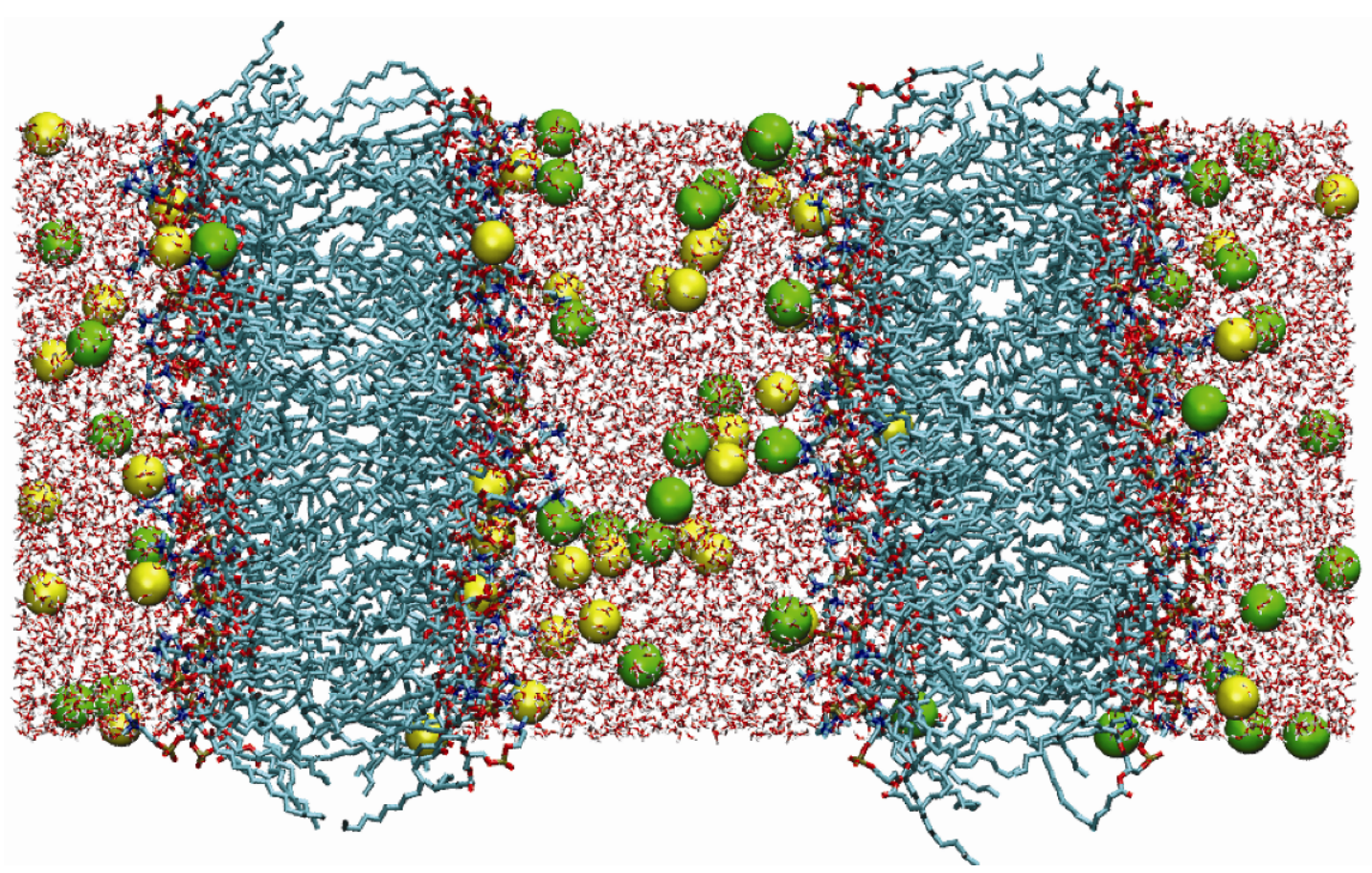

Figure 5.1. Initial structure of a double-bilayer system with a charge imbalance of $6 \mathrm{Na}^{+}$ions per bilayer. The sodium ion concentration is larger in the "inner" water bath between two DMPC bilayers (see text for details). The two lipid and water regions are complemented by ions; sodium ions in light and chloride ions with darker color. Adapted with permission from [34]. Copyright $\odot$ 2007, American Chemical Society. Please visit http://www.springer.com/series/7845 to view a high-resolution full-color version of this illustration.

In a double-bilayer system, the two bilayers separate the "inner" (between the bilayers) and "outer" water reservoirs. Here, the terms "inner" and "outer" are used for convenience only, as periodic boundary conditions are applied in all three directions. The next step is to create a transmembrane ionic charge imbalance. To do that, cations $\left(\mathrm{Na}^{+}\right.$or $\mathrm{K}^{+}$ions $)$and anions $\left(\mathrm{Cl}^{-}\right.$ ions) are added to the system, replacing randomly chosen water molecules. The number of anions is set to be the same in both water reservoirs $\left(20 \mathrm{Cl}^{-}\right.$ions). Meanwhile, an excess of cations is created in the "inner" water phase with respect to the "outer" one. The initial transmembrane imbalance of cations is chosen to be 6 cations per bilayer or 12 cations between the reservoirs, such that 26 and 14 cations are placed in the "inner" and "outer" water reservoirs, respectively (see Fig. 5.1). The condition of electroneutrality in the whole double-bilayer system is fulfilled.

To gain insight into characteristic times of pore formation and sensitivity of the whole process to the type of salt, 20 different runs simulated over 10 to $15 \mathrm{~ns}$ each have been performed by varying initial conditions (10 runs with $\mathrm{NaCl}$ and 10 runs with $\mathrm{KCl}$ ) [10,11]. Figure 5.2 depicts the transmembrane electric field and electrostatic potential for one representative double-bilayer system with $\mathrm{NaCl}$. We find that for this particular system the imbalance of $6 \mathrm{Na}^{+}$ions per bilayer induces on average a transmembrane voltage of about $2.12 \mathrm{~V}$ across the membrane. 


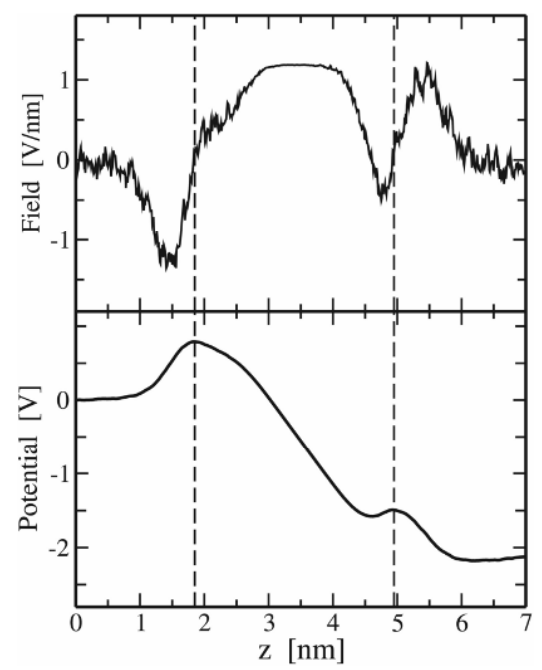

Figure 5.2. Electric field (top) and electrostatic potential (bottom) versus distance $z$ from the middle of the "inner" water phase between the two bilayers. The field and the potential were calculated from the Poisson equation by integrating over charge densities that were determined directly from MD simulations; both quantities were chosen to be zero in the middle of the "inner" water phase $(z=0)$. Only the initial part of the trajectory prior to actual pore formation was used for the calculation. Dashed lines indicate average positions of phosphorous atoms of lipids for the two bilayer leaflets. Reprinted with permission from [11]. Copyright (C) 2007, The Biophysical Society.

Remarkably, the applied transmembrane imbalance of sodium ions gives rise to the formation of a water pore, which starts about $1.4 \mathrm{~ns}$ after the beginning of the simulation. The various stages of pore formation can be readily followed by considering water molecules in the hydrophobic core of a membrane (Figs. 5.3 and 5.4). The first step in pore formation is the creation of a single water defect spanning the entire membrane (Fig. 5.4B); this "chain" of water molecules expands rapidly on a nanosecond timescale (see Figs. 5.3 and 5.4C). The expansion of the pore is accompanied by a considerable redistribution of lipid headgroups from the water-lipid interface to the membrane interior; the hydrophilic headgroups line the pore and stabilize it. It is also interesting to note that the simulations did not express any signs of simultaneous formation of multiple pores. Finally, after most of the excess ions have migrated across the pore, the transmembrane field has become too weak to maintain the pore, which hence becomes metastable. Nonetheless, despite its metastable nature, the pore may remain open for a reasonably long period of time (see below).

The overall molecular picture of pore formation found here is very similar to the picture reported in MD simulations of electroporation in lipid membranes under the influence of an external (constant) electric field [35-37]. This similarity is due to the fact that in both situations a lipid membrane is exposed to a rather high transmembrane electric field. It should be emphasized that the electric field induced by a transmembrane ionic density gradient in the present case is stochastic in nature: the transmembrane electric field is determined by instantaneous positions of ions and is therefore subject to considerable fluctuations in time and space. Indeed, it has been found [11] that the average values of the electric field across a membrane (prior to pore formation) can scatter from 1.74 to $3.33 \mathrm{~V}$. 


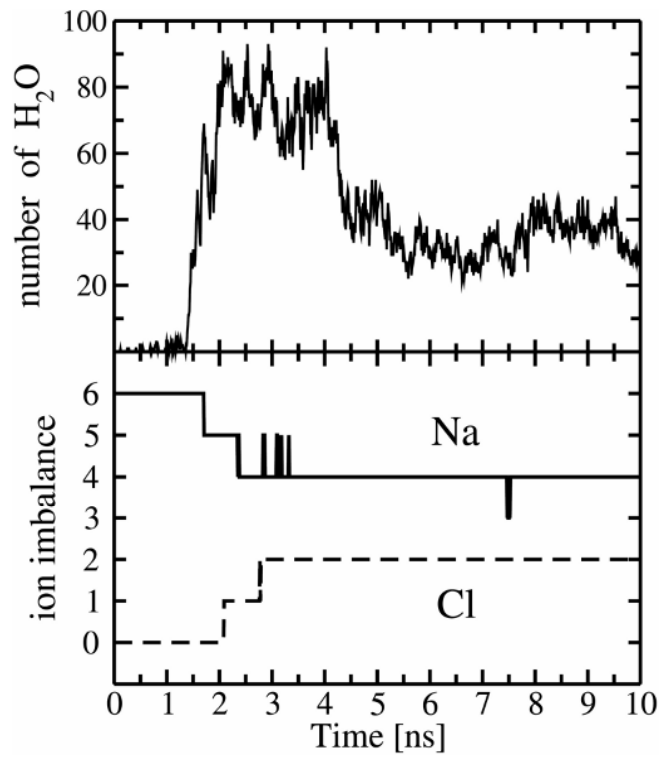

Figure 5.3. Time evolution for the number of water molecules in a pore. The number of water molecules is computed within $0.5 \mathrm{~nm}$ from the bilayer center (top) and for the transmembrane imbalance of $\mathrm{Na}^{+}$and $\mathrm{Cl}^{-}$ions (bottom). Reprinted with permission from [11]. Copyright (C) 2007, The Biophysical Society.

The transmembrane potential of this magnitude exceeds the potentials typical for native plasma membranes by one order of magnitude and thus deserves some discussion. Due to obvious limitations of atomic-scale MD simulations regarding the length and timescales accessible, one has to promote pore formation by increasing the ionic charge imbalance across a membrane and, correspondingly, the transmembrane potential. By doing so, one essentially optimizes chances for observing the formation of water pores on timescales that are within reach by atomistic simulations. A similar approach has been used in simulations that mimic electroporation [35-37]. The choice of a reasonably large ion concentration gradient for simulation studies is also supported by the nature of biological matter, as it is driven by thermal fluctuations: in a macroscopic system, density fluctuations inevitably lead to fluctuations of ion densities that may locally be considerably larger than the average ones. Moving on, it is noteworthy that the overall picture of pore formation seems to be generic in the sense that the same stages of pore formation have also been found for a considerably smaller ion concentration gradient, though the characteristic timescales are then obviously longer [11]. On these grounds, it is reasonable to assume that the same pore formation mechanism would be found also with an ion imbalance that would match conditions in native cells.

The maximum size of a transient water pore varies considerably from one simulation to another. Assuming an ideal cylindrical shape for a pore, the radius of the inner part of a pore varies from 0.41 to $1.03 \mathrm{~nm}$. Essentially, after the water pore has become large enough, the cations and anions permeate through the pore along the ion density gradient (cations and anions permeate in opposite directions). As an example, Figures 5.3 and 5.4 show permeation of $\mathrm{Na}$ and $\mathrm{Cl}$ ions across the membrane, discharging the transmembrane potential and driving the system to 

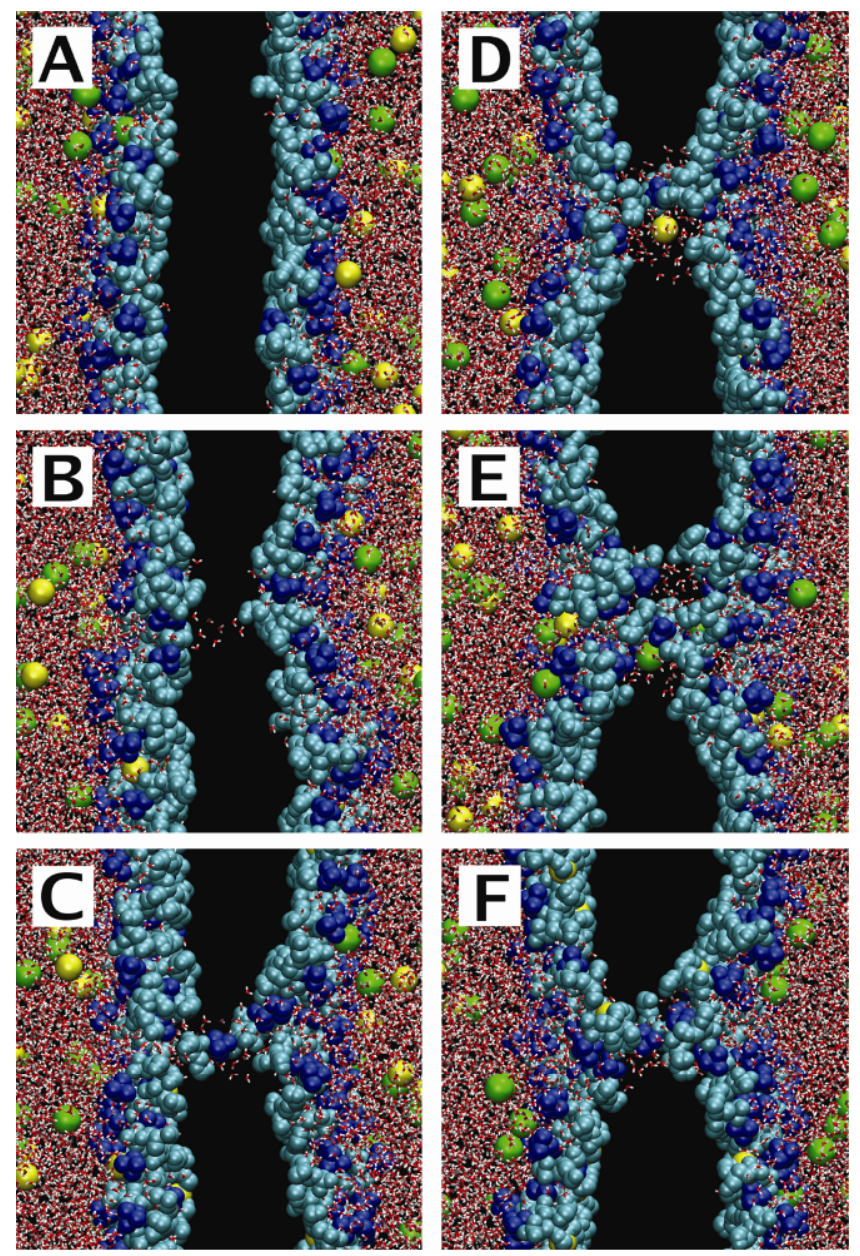

Figure 5.4. Pore formation and pore-mediated ion transport induced by a transmembrane imbalance of sodium ions. (A) 0 ps, the initial structure; (B) 1420 ps, appearance of a single water defect; (C) 1490 ps, redistribution of lipid headgroups toward the membrane interior; (D) $1710 \mathrm{ps}$, leakage of a sodium ion; (E) $2090 \mathrm{ps}$, leakage of a chloride ion; and (F) $10 \mathrm{~ns}$, the metastable state of the water pore. Water is shown on the left- and right-hand sides of the figures, choline groups of lipid headgroups in dark, phosphate and glycerol groups using lighter color in the same region, and $\mathrm{Na}^{+}\left(\mathrm{Cl}^{-}\right)$ions as light (dark) spheres. Nonpolar acyl chains of lipids are not shown. Excess of $\mathrm{Na}^{+}$ions is on the left-hand side. Reprinted with permission from [11]. Copyright (C) 2007, The Biophysical Society. The figure is adapted from (34). Please visit http://www.springer.com/series/7845 to view a high-resolution full-color version of this illustration.

equilibrium. It turns out that the size of the pore is controlled by the induced transmembrane potential: the transmembrane ionic leakage leads to a remarkable drop in the transmembrane potential difference and, as a result, to a considerable decrease in pore size (see Figs. 5.3 and 5.4). The drop in the ionic charge imbalance from 6 to 2 within the first $5 \mathrm{~ns}$ of simulations gives rise to a decrease in the number of water molecules by about $70 \%$ compared to its maximum value. In general, after a drop of the transmembrane voltage below some critical value due to ion leakage, the water pore becomes metastable, its lifetime expressing a broad distribution from 5 to more than $200 \mathrm{~ns}$. 
It is interesting to compare how the molecular picture of pore formation and subsequent ion leakage depend on the type of salt ions used to create a transmembrane ionic charge imbalance. It turns out that the sequence of events and most of the features of pore formation are very similar to that of $\mathrm{NaCl}$ and $\mathrm{KCl}$ salts; the maximal radius of the induced pores has been observed to be somewhat smaller in the case of potassium ions, as they require smaller pores for permeation to occur. However, ionic leakage through the pore is found to be sensitive to the type of cation: $\mathrm{K}^{+}$ions permeate through a lipid membrane much easier than $\mathrm{Na}^{+}$ions do. The origin of such a difference is most likely in the strong interactions of sodium ions with lipid headgroups lining the pore walls [43-47]. In contrast, potassium ions interact only weakly with the carbonyl regions of phospholipids [11,47].

\subsection{FLIP-FLOPS OF LIPID MOLECULES ACROSS PROTEIN-FREE LIPID MEMBRANES}

Given the above findings for pore formation, it is tempting to ask whether the pores could act as membrane defects, facilitating the occurrence of flip-flops. To consider this scenario, let us highlight some recent data [34] for 8 double-bilayer systems with preformed pores (4 systems with $\mathrm{NaCl}$ as well as $\mathrm{KCl}$ ) that were simulated over $200 \mathrm{~ns}$, or until the transient water pore closed.

In all the considered systems, one finds spontaneous pore-mediated translocation processes of lipid molecules from one leaflet to another. The overall process of lipid flip-flop is depicted in Figure 5.5 for one particular lipid of a DMPC bilayer system with $\mathrm{NaCl}$. Starting from an intact lipid membrane (Fig. 5.5A), one first observes the formation of a water pore spanning the entire membrane. For the tagged lipid studied here, the pore is laterally located quite far away from the lipid (Fig. 5.5B), implying that a flip-flop cannot occur right after pore formation. However, after about $100 \mathrm{~ns}$ the lipid molecule has diffused laterally to the vicinity of the pore, allowing it to enter the pore and become part of it as the lipid's headgroup settles to line the pore (Fig. 5.5C). At this point, the spontaneous translocation of the lipid through the pore initiates: the translocation involves diffusion of a lipid headgroup along the pore walls coupled to the simultaneous desorption of lipid acyl chains out of the bilayer leaflet (Fig. 5.5D). As a result, one observes the appearance of the lipid in the opposite bilayer leaflet, accompanied by progressive reorientation of the lipid (Fig. 5.5E). For translocation to be successfully accomplished, the lipid should be irreversibly accommodated in the opposite leaflet. It turns out that such accommodation can be a rather slow process. For instance, more than $50 \mathrm{~ns}$ are required for the particular flip-flopped lipid considered here to settle down in the opposite leaflet (see Fig. 5.5EG). Considering that the actual flip-flop takes about 30 to $100 \mathrm{~ns}$ to occur, the timescale of settling down is rather short and essentially limited by lateral diffusion: to avoid being displaced back to the original leaflet, the headgroup of the lipid being translocated should first be able to detach its headgroup out of pore "walls," followed by lateral diffusion of a lipid away from the pore site. Since diffusion in the plane of fluid membranes over a scale of molecular size takes roughly about $20 \mathrm{~ns}$, it is obvious that about $50 \mathrm{~ns}$ are required to diffuse away from the immediate vicinity of the pore. We note, however, that the realization of a flip-flop can be facilitated by the closure of a pore; for the particular system in question, pore closure occurs at $t \cong 210 \mathrm{~ns}$ (Fig. 5.5H). 

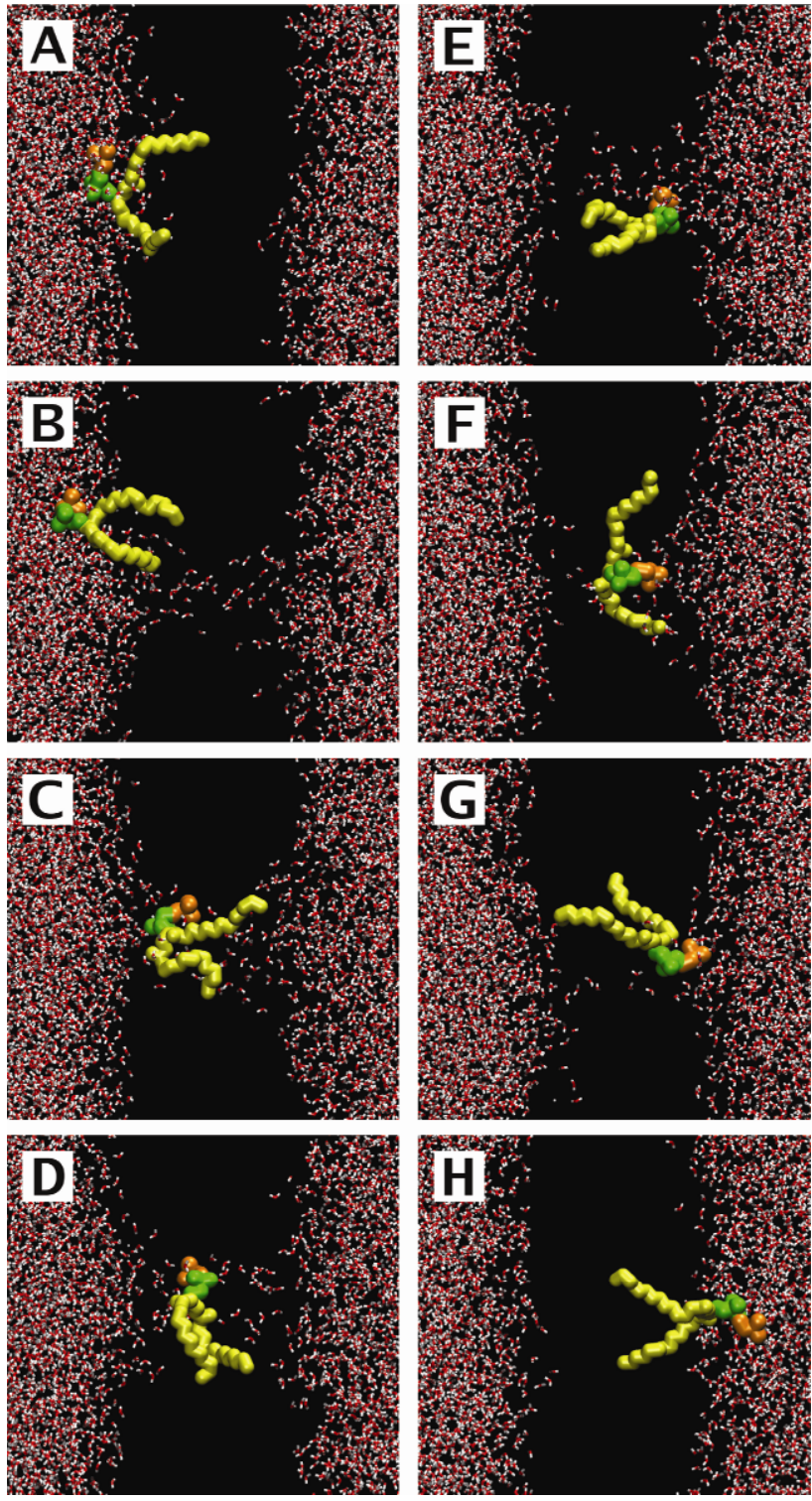

Figure 5.5. Pore-mediated lipid translocation across a DMPC membrane in aqueous solution with NaCl. (A) 0 ns, (B) 43.85 ns, (C) 118.9 ns, (D) 122.4 ns, (E) 152.7 ns, (F) 204.65 ns, (G) 208.9 ns, and (H) 215 ns. Lipids (except for the flip-flopped one) and ions are not shown; water is shown on the left- and right-hand sides of the figures, and the flip-flopped lipid is shown separately during various stages of the process. Reprinted with permission from [34]. Copyright (C) 2007, American Chemical Society. Please visit http://www.springer.com/series/7845 to view a high-resolution full-color version of this illustration.

To visualize lipid flip-flops across a membrane, it is instructive to follow the time evolution of the positions of centers of mass (CM) of lipid headgroups as well as the lipids' overall orientation measured through the angle between the bilayer normal and the vector directed from the 

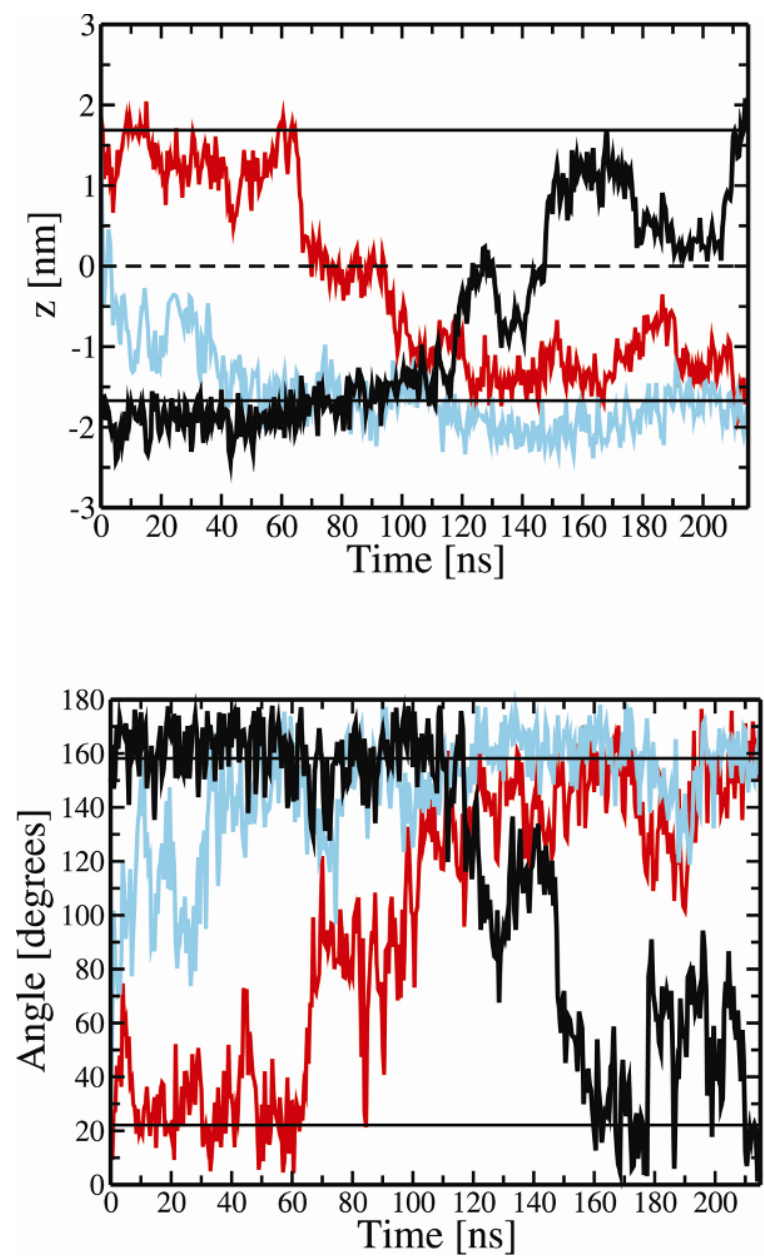

Figure 5.6. Time evolution of lipid positions and their orientations. Top: Time evolution of positions of the centers of mass (CM) of headgroups for four representative flip-flopped lipids of a DMPC bilayer system in an aqueous solution with $\mathrm{NaCl}$. The $z=0$ corresponds to the membrane center; solid black lines show the average positions of lipid headgroups in the two opposite leaflets, extracted from the intact membrane before pore formation. Bottom: Time evolution of the tail-to-head orientation for the same four flipflopped lipids. The tail-to-head orientation is characterized as the angle between the bilayer normal and the vector directed from the CM of acyl chains to the CM of headgroups. Solid black lines again show typical values for the angle for lipids in the opposite leaflets. Adapted with permission from [34]. Copyright (C) 2007, American Chemical Society. Please visit http://www.springer.com/series/7845 to view a highresolution full-color version of this illustration.

$\mathrm{CM}$ of lipid's hydrocarbon chains to the $\mathrm{CM}$ of its headgroup. Figure 5.6 presents trajectories for four representative flip-flopped lipids of a DMPC bilayer with $\mathrm{NaCl}$ [34]. Remarkably, the translocation of a lipid molecule across a membrane closely correlates with the overall reorientation of the lipid within a membrane: when a lipid molecule is accommodated in its initial membrane leaflet, the lipid's tail-to-head vector makes an average angle of 22 degrees (or 158 degrees depending on the leaflet) with the bilayer normal. During flip-flop (i.e., when the CM of a lipid headgroup crosses the membrane center, $z=0$ ), a lipid changes its orientation and the 
corresponding angle lies between the two values typical to opposite leaflets. Interestingly, lipids tend to adopt an orientation perpendicular to the bilayer normal when they become close to the membrane center, which is clear for one of the lipids in Figure 5.6.

One can clearly distinguish two different types of lipid translocations [34]:

1. Flip-flops of lipid molecules that are directly involved in the initial formation of a water pore. These are very fast flip-flop processes whose onset coincides with the formation of a pore. They are also characterized by short times (10-20 ns) required for accommodation in the opposite leaflet (see the light curve in Fig. 5.6).

2. Considerably slower flip-flops of lipids that are either involved in initial pore formation but require much longer times to accomplish translocation (the dark curve in Fig. 5.6) or are initially remote from the pore site but diffuse to the pore with time (remaining curves in Fig. 5.6).

Overall, out of 50 successful flip-flop events observed, only 9 flip-flops are associated with category 1 . The rest of the flip-flops (about $80 \%$ ) belong to category 2 . On the basis of these 50 flip-flop events resolved in atomic detail, one can estimate that the average time required for a lipid to translocate through a preformed water pore in a membrane is around $60 \mathrm{~ns}$. Yet there is reason to mention that flip-flop times for individual lipids scatter considerably and have here been found to range from 10 to $130 \mathrm{~ns}$. The reasonable statistics resulting from these events allows one to present the distribution of these flip-flop times (see Fig. 5.7). First, one finds that the distribution is indeed very wide. Second, one can easily distinguish two peaks that correspond to the two flip-flop categories outlined above: there is a clear peak around $t \cong 20 \mathrm{~ns}$ (flipflop category 1), a rather broad peak around the average flip-flop time $t \cong 60 \mathrm{~ns}$ (category 2), and a long tail extending up to $t=130 \mathrm{~ns}$ corresponding to the slowest flip-flop observed.

Of the factors that affect the rate of lipid translocation, let us mention pore opening and membrane resealing. As discussed above, pore formation implies fast reorientation of some lipid headgroups toward the membrane interior [10,11,35-37] so that the lipids involved in pore formation are driven off from their equilibrium state. If desorption of lipid hydrocarbon chains occurs at the same time, one can observe a very fast lipid flip-flop. However, in most cases this scenario takes place rather rarely (see Fig. 5.7), because the time required for lipid accommodation in the opposite leaflet can be considerable. Pore closure, in turn, considerably speeds up the flip-flops of lipids that are partly translocated since irreversible membrane resealing simply makes it impossible for the lipids to move back to their initial leaflet. As an example, one can mention the translocation of a lipid shown by the dark curve in Figure 5.6 (note that pore closure for this bilayer system occurs at $t \cong 210 \mathrm{~ns}$ ).

Another factor whose role deserves some discussion is the type of cations used for pore formation. All together, 18 successful lipid flip-flops were found for DMPC bilayers with $\mathrm{NaCl}$, while for their counterparts with $\mathrm{KCl}$ a number of 32 pore-mediated lipid translocations were observed. Therefore, despite the fact that the average times required for lipid flip-flops are similar in both cases (64 and $60 \mathrm{~ns}$ for bilayers with $\mathrm{NaCl}$ and $\mathrm{KCl}$ salt, respectively), the type of salt used does affect the probability of lipid flip-flop. In more practical terms, DMPC bilayer systems with $\mathrm{NaCl}$ and $\mathrm{KCl}$ were simulated for 485 and $515 \mathrm{~ns}$, respectively; yet, the overall number of lipid flip-flops was almost twice as large in the case of $\mathrm{KCl}$ (see also Fig. 5.7). One can conclude that the probability of a spontaneous flip-flop event in a bilayer system with potassium ions is higher by a factor of about 1.7. This finding can readily be explained in terms of 
interactions of cations with lipid headgroups: sodium ions demonstrate much stronger interactions with zwitterionic phosphatidylcholine lipids than potassium ions [11,47]. More specifically, sodium ions are known to bind strongly to carbonyl oxygens of phosphatidylcholines, leading to the formation of tight complexes between neighboring lipids [11,43-47], thus weakening the desorption of lipids out of membrane leaflets toward the membrane interior.

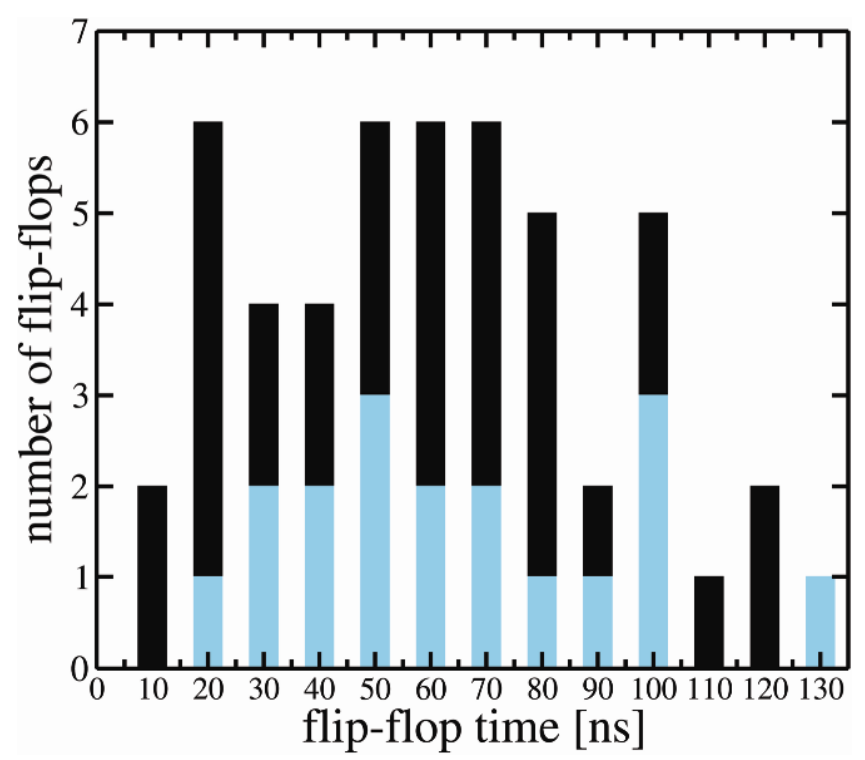

Figure 5.7. The distribution of flip-flop times. The distribution is shown for all eight DMPC bilayer systems considered (50 flip-flop events all together). The lipid flip-flops in the bilayer systems with $\mathrm{NaCl}$ are shown in light color, while the rest were observed under the influence of $\mathrm{KCl}$. Adapted with permission from [34]. Copyright () 2007, American Chemical Society. Please visit http://www.springer.com/series/7845 to view a high-resolution full-color version of this illustration.

Overall, the molecular mechanism of lipid flip-flop discussed here consists of two steps [34]. The first step is the formation of a transient water defect in a membrane. In the present case this is achieved through the incorporation of an initial ionic concentration imbalance across the membrane; such an imbalance, being an inherent feature of plasma membranes of eukaryotic cells, initiates the formation of a water pore coupled to the subsequent fast ion leakage that quickly reduces the local field strength and makes the pore metastable. The second step is a fast translocation of lipid molecules through preformed water pores. Because lipid translocation through a pore is very fast (taking only $60 \mathrm{~ns}$ on average), one can observe considerable mixing of lipids between the opposite leaflets since the lifetime of water pores ranges from tens to several hundreds of nanoseconds (see Fig. 5.8).

The origin of the force that drives the pore-mediated lipid translocation across a membrane is central for understanding the molecular mechanism of lipid flip-flop. First, we recall that the PC lipids considered in this study are zwitterionic, i.e., neutral. Therefore, the transmembrane electrostatic potential can hardly play the main role. Indeed, the membrane-normal component of the total electrostatic force exerted on the lipids involved in flip-flops is found to fluctuate 
around zero [34], without a component that would drive translocation. Second, as already discussed, the electric field across a membrane is spatially inhomogeneous, as it is defined by instantaneous positions of ions. In general, the inhomogeneous electric field could contribute to translocation of zwitterionic lipids via dielectrophoretic motion. However, it has been found that the contribution of the dielectrophoretic force exerted on the dipolar lipid headgroup is the largest for a small number of lipids involved in flip-flops during the initial stages of pore formation, and even then the dielectrophoretic component is comparable to thermal energy [34]. At longer times, when the transmembrane electric field is almost fully discharged due to ion leakage, the dielectrophoretic force has been found to be an order of magnitude smaller than the force due to thermal fluctuations. Thus, we conclude that translocation of lipids through transient water pores is mainly diffusive, i.e., driven by thermal fluctuations.
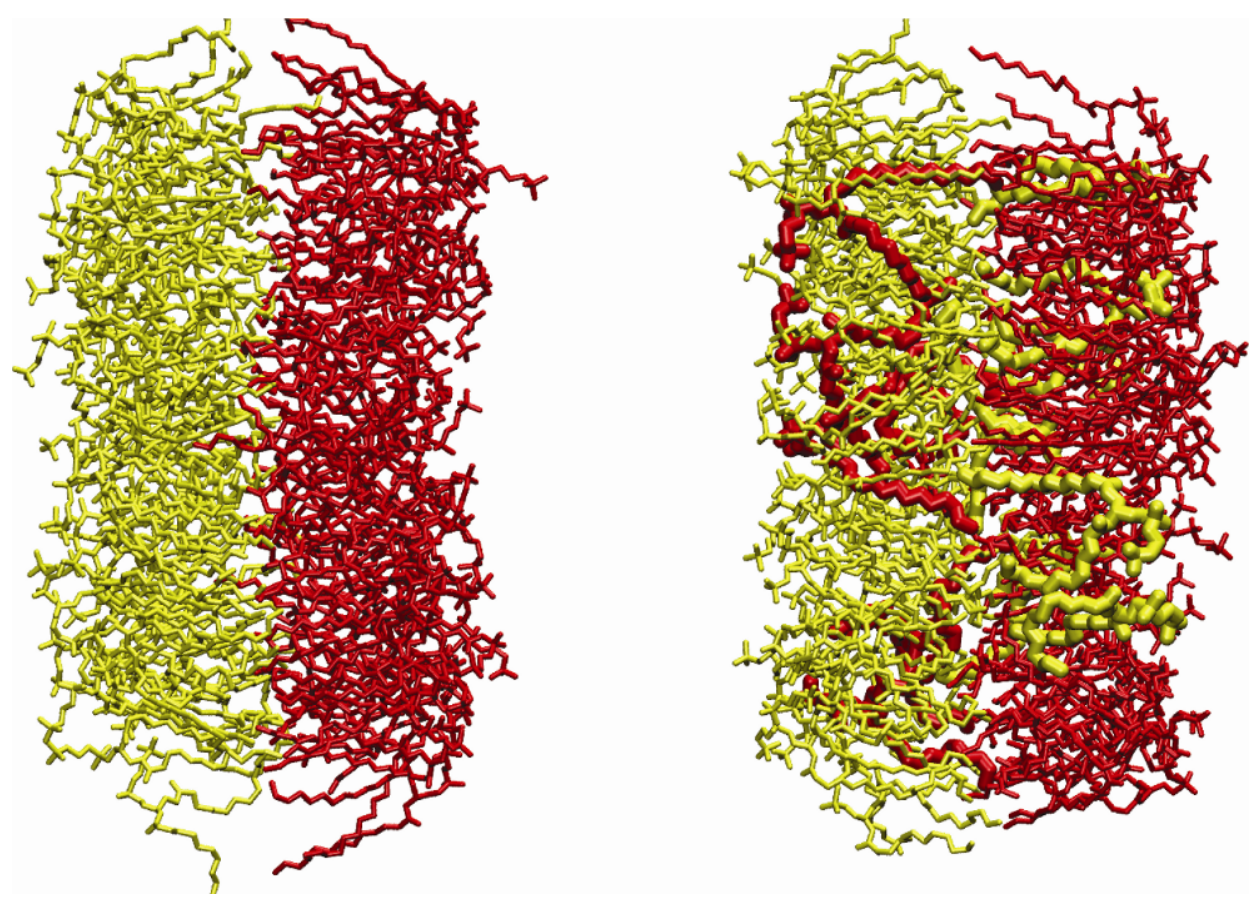

Figure 5.8. Flip-flop induced mixing up of the lipids which belong to the opposite leaflets of a DMPC bilayer system with KCl. Left: Intact membrane at $t=0 \mathrm{~ns}$. Right: The same membrane with an opened pore at $t=200 \mathrm{~ns}$. Lipids in opposite leaflets are shown in light and dark; for clarity, the size of flip-flopped lipids has been enlarged. Adapted with permission from [34]. Copyright (C) 2007, American Chemical Society. Please visit http://www.springer.com/series/7845 to view a high-resolution full-color version of this illustration.

Overall, the atomic-scale MD simulations demonstrate that the appearance of a water pore spanning a phospholipid membrane inevitably leads to diffusive transmembrane translocation of lipids through the pore. There is reason to especially emphasize that, while the formation of a water pore is induced here by a transmembrane ion density gradient, the precise way by which the pore is formed is irrelevant for the flip-flop mechanism discussed here. This has also been 
confirmed by very recent studies of chemically induced pore formation in phospholipid membranes: it was demonstrated that a small amphiphilic solute dimethylsulfoxide (DMSO) which is widely employed in cell biology as an effective penetration enhancer [48], cryoprotectant [49], and cell fusogen [50] — is able to induce hydrophilic water pores in protein-free lipid membranes [51-53]. What is more, the appearance of DMSO-induced pores (formed without salt ions) was shown to lead to spontaneous pore-mediated lipid translocations [54], indicating that the formation of a water pore spanning the membrane is most likely the only required prerequisite for transmembrane lipid flip-flop.

Moving on, the above-discussed results provide concrete evidence for a view that the formation of water pores (defects) is the rate-limiting step in the lipid flip-flop processes that take place through pores. While the actual flip-flops driven by thermal fluctuations are very rapid processes, occurring spontaneously on a timescale of tens of nanoseconds, the total timescales of lipid flip-flops range from minutes to hours. The rate-limiting step is hence pore formation. Recent simulation studies and free energy calculations are in favor of this view $[33,55,56]$.

However, we have to keep in mind that the above-discussed scheme is not necessarily the only possible mechanism for lipid translocation. Sterols have been observed to translocate without pores [57,58]. On the other hand, sterols are known to translocate at much larger rates compared to many other lipids, the timescales ranging over seconds rather than hours, which may explain why transient pores are not crucial for their translocation processes. Also, there is evidence for flip-flops that take place without transient membrane defects. The conditions where this has been reported to occur include high concentrations of glucose and a high transmembrane $\mathrm{pH}$ gradient [59].

Nonetheless, coming back to pore formation, let us summarize that the rate of lipid flipflops can be significantly enhanced by spontaneous formation of water pores. In addition to their biological relevance, this view also offers a molecular-level framework for the development of ways to promote lipid transmembrane translocation and to make it possible to manipulate lipid distribution across cell membranes. This view also provides an explanation for the experimentally observed fact that the exposure of lipid membranes to electric field pulses drastically reduces the timescales where flip-flops take place [60]. Strong external electric fields induce electroporation of a lipid membrane, considerably increasing the number of pathways (defects) suitable for pore-mediated lipid flip-flops. Similarly, the presented pore-mediated mechanism for lipid flip-flops can be invoked to shed more light on how local anesthetics, amphiphilic compounds, and pore-forming peptides facilitate the occurrence of flip-flops [59].

\subsection{SUMMARY}

The results presented and discussed in this chapter illustrate the importance of understanding the complex interplay of lipids with water and ions. The dynamical processes associated with these systems are collective, involving a large number of molecules to function in unison. Pore formation and lipid flip-flops are excellent examples of this fact. Nonetheless, they are just a minor scratch on the surface of the issue since there are numerous membrane processes where the same view holds, too. For example, the fusion of membranes and endocytosis as well as exocytosis are large-scale processes that involve large amounts of lipids, whose joint dynamic interplay leads to realization of these events. Further, it has been shown very recently that lipid 
diffusion in the membrane plane occurs through the diffusion of locally correlated transient lipid clusters that move in unison, and the dynamical correlations associated with these motions range over tens of nanometers [61]. Also, as membranes are soft interfaces driven by weak interactions of the order of $k_{\mathrm{B}} T$, it is not particularly surprising that membranes are characterized by undulations that are essentially collective motions of lipids in the bilayer normal direction. The elastic behavior of membranes exemplified by undulations also has a significant role to play in membrane protein functions, since it has been shown that the functionality of certain classes of proteins depends on elastic coefficients such as bending rigidity and area compressibility [62,63]. Concluding, the dynamics of lipids in membranes is an exceptionally appealing example of the dynamics of soft matter, and the collective nature of lipid motions has to be always accounted for if one desires to understand the physical laws that govern membrane dynamics.

\section{ACKNOWLEDGMENTS}

This work has been supported by the Russian Foundation of Basic Research through Grant No. 08-03-00150 (A.A.G.) and by the Academy of Finland (I.V.). The simulations were performed at the Finnish IT Center for Science and on the HorseShoe (DCSC) supercluster at the University of Southern Denmark.

\section{PROBLEMS}

5.1. In the chapter we discussed the overall process of pore-mediated lipid flip-flops and illustrated it for a dimyristoylphosphatidylcholine (DMPC) bilayer at $T=323 \mathrm{~K}$. Make qualitative predictions regarding changes in the flip-flop rate when (a) temperature is decreased to e.g. the physiological value $(T=310 \mathrm{~K})$; (b) DMPC lipids are replaced by dipalmitoylphosphatidylcholine (DPPC) lipids whose acyl chains are two hydrocarbons longer.

5.2. Assuming that the lipid lateral diffusion coefficient is $D=1 \times 10^{-7} \mathrm{~cm}^{2} / \mathrm{s}$, compute the time that is needed to diffuse from one side of a cell to the opposing one, assuming the cell radius to be $\mathrm{R}=10$ micrometers.

\section{FURTHER READING}

For further reading on transmembrane translocations of lipids in cell membranes, one can recommend several recent review papers that summarize a contemporary view of related phenomena [26,64-67]. A thorough account of experimental observations of chemically induced lipid flip-flops (both pore-mediated ones and those that do not involve pores) can be found in [59]. A very recent computational study additionally highlights the overall process of pore formation in phospholipid membranes at atomistic resolution [68]. 


\section{REFERENCES}

1. Mouritsen, OG. 2005. Life - as a matter of fat. Berlin: Springer-Verlag.

2. Katsaras J, Gutberlet T, eds. 2001. Lipid bilayers: structure and interactions. Berlin: Springer-Verlag.

3. Yeagle PL, ed. 2005. The structure of biological membranes. Boca Raton, FL: CRC Press.

4. Simons K, Ikonen, E. 1997. Functional rafts in cell membranes. Nature 387:569-572.

5. Edidin M. 2003. The state of lipid rafts: from model membranes to cells. Annu Rev Biophys Biomol Struct 32:257-283.

6. Pike LJ. 2004. Lipid rafts: heterogeneity on the high seas. Biochem J 378:281-292.

7. Hancock JF. 2006. Lipid rafts: continuous only from simplistic standpoints. Nat Rev Mol Cell Biol 7:456-462.

8. Saxton MJ, Jacobson K. 1997. Single-particle tracking: applications to membrane dynamics. Annu Rev Biophys Biomol Struct 26:373-399.

9. Vattulainen I, Mouritsen OG. 2005 Diffusion in membranes. In Diffusion in condensed matter: methods, materials, models, pp. 471-509. Ed P Heithans, J Kärger. Berlin: Springer-Verlag.

10. Gurtovenko AA, Vattulainen I. 2005. Pore formation coupled to ion transport through lipid membranes as induced by transmembrane ionic charge imbalance: atomistic molecular dynamics study. J Am Chem Soc 127: $17570-17571$.

11. Gurtovenko AA, Vattulainen I. 2007. Ion leakage through transient water pores in protein-free lipid membranes driven by transmembrane ionic charge imbalance. Biophys J 92:1878-1890.

12. Feller SE, Gawrisch K, MacKerell Jr AD. 2001. Polyunsaturated fatty acids in lipid bilayers: intrinsic and environmental contributions to their unique physical properties. J Am Chem Soc 124:318-326.

13. Doxastakis M, Sum AK, de Pablo JJ. 2005. Modulating membrane properties: the effect of trehalose and cholesterol on a phospholipid bilayer. J Phys Chem B 109:24173-24181.

14. Klauda JB, Roberts MF, Redfield AG, Brooks BR, Pastor RW. 2008. Rotation of lipids in membranes: molecular dynamics simulation, 31P spin-lattice relaxation, and rigid-body dynamics. Biophys J 94:3074-3083.

15. Moore PB, Lopez CF, Klein ML. 2001. Dynamical properties of a hydrated lipid bilayer from a multinanosecond molecular dynamics simulation. Biophys J 81:2484-2494.

16. Niemelä P, Ollila S, Hyvönen MT, Karttunen M, Vattulainen I. 2007. Assessing the nature of lipid raft membranes. PLoS Comput Biol 3:304-312.

17. Filippov A, Orädd G, Lindblom G. 2006. Sphingomyelin structure influences the lateral diffusion and raft formation in lipid bilayers. Biophys J 90:2086-2092.

18. Gennis GB. 1989. Biomembranes: molecular structure and function. New York: Springer-Verlag.

19. Zachowski A. 1993. Phospholipids in animal eukaryotic membranes: transverse asymmetry and movement. Biochem J 294:1-14.

20. Manno S, Takakuwa Y, Mohandas N. 2002. Identification of a functional role for lipid asymmetry in biological membranes: phosphatidylserine-skeletal protein interactions modulate membrane stability. Proc Natl Acad Sci USA 99:1943-1948.

21. Latorre R, Hall JE. 1976. Dipole potential measurements in asymmetric membranes. Nature 264:361-363.

22. Gurtovenko AA, Vattulainen I. 2007. Lipid transmembrane asymmetry and intrinsic membrane potential: two sides of the same coin. J Am Chem Soc 129:5358-5359.

23. Gurtovenko AA, Vattulainen I. 2008. Membrane potential and electrostatics of phospholipid bilayers with asymmetric transmembrane distribution of anionic lipids. J Phys Chem B 112:4629-4634.

24. Pomorski T, Hrafnsdottir S, Devaux PF, van Meer G. 2001. Lipid distribution and transport across cellular membranes. Semin Cell Dev Biol 12:139-148.

25. Balasubramanian K, Schroit AJ. 2003. Aminophospholipid asymmetry: a matter of life and death. Annu Rev Physiol 65:701-734.

26. Bevers EM, Comfurius P, Dekkers DWC, Zwaal RFA. 1999. Lipid translocation across the plasma membrane of mammalian cells. Biochim Biophys Acta 1439:317-330.

27. Pomorski T, Menon AK. 2006. Lipid flippases and their biological functions. Cell Mol Life Sci 63:2908-2921.

28. Raggers RJ, Pomorski T, Holthuis JCM, Kälin N, van Meer G. 2000. Lipid traffic: the ABC of transbilayer movement. Traffic 1:226-234.

29. Holthuis JC, Levine TP. 2005. Lipid traffic: floppy drives and a superhighway. Nat Rev Mol Cell Biol 6:209_ 220. 
30. Kornberg RD, McConnell HM. 1971. Inside-outside transition of phospholipids in vesicle membranes. Biochemistry 10:1111-1120.

31. Wimley WC, Thompson TE. 1990. Exchange and flip-flop of dimyristoylphosphatidylcholine in liquidcrystalline, gel, and two-component, two-phase large unilamellar vesicles. Biochemistry 29:1296-1303.

32. Schwarz S, Haest CWM, Deuticke B. 1999. Extensive electroporation abolishes experimentally induced shape transformations of erythrocytes: a consequence of phospholipids symmetrization? Biochim Biophys Acta 1421:361-379.

33. Tieleman DP, Marrink SJ. 2006. Lipids out of equilibrium: Energetics of desorption and pore mediated flip-flop. J Am Chem Soc 128:12462-12467.

34. Gurtovenko AA, Vattulainen I. 2007. Molecular mechanism of lipid flip-flop. J Phys Chem B 111:1355413559.

35. Tieleman DP, Leotiadou H, Mark AE, Marrink SJ. 2003. Simulation of pore formation in lipid bilayers by mechanical stress and electric fields. J Am Chem Soc 125:6382-6383.

36. Tieleman DP. 2004. The molecular basis for electroporation. BMC Biochemistry 5:10.

37. Tarek M. 2005. Membrane electroporation: a molecular dynamics simulation. Biophys J 88:4045-4053.

38. Leontiadou H, Mark AE, Marrink SJ. 2004. Molecular dynamics simulations of hydrophilic pores in lipid bilayers. Biophys J 86:2156-2164.

39. Leontiadou H, Mark AE, Marrink SJ. 2007. Ion transport across transmembrane pores. Biophys J 92:4209-4215.

40. Voet D, Voet JG. 2004. Biochemistry, 3rd ed. New York: John Wiley \& Sons.

41. Cevc G, Marsh D. 1987. Phospholipid bilayers: physical principles and models. New York: John Wiley \& Sons.

42. Sachs JN, Crozier PS, Woolf TB. 2004. Atomistic simulations of biologically realistic transmembrane potential gradients. J Chem Phys 121:10847-10851.

43. Gurtovenko AA. 2005. Asymmetry of lipid bilayers induced by monovalent salt: atomistic molecular dynamics study. J Chem Phys 122:244902.

44. Böckmann RA, Hac A, Heimburg T, Grubmüller H. 2003. Effect of sodium chloride on a lipid bilayer. Biophys J 85:1647-1655.

45. Pandit SA, Bostick D, Berkowitz ML. 2003. Molecular dynamics simulation of a dipalmitoylphosphatidylcholine bilayer with $\mathrm{NaCl}$. Biophys J 84:3743-3750.

46. Gurtovenko AA, Miettinen M, Karttunen M, Vattulainen I. 2005. Effect of monovalent salt on cationic lipid membranes as revealed by molecular dynamics simulations. J Phys Chem B 109:21126-21134.

47. Gurtovenko AA, Vattulainen I. 2008. Effect of $\mathrm{NaCl}$ and $\mathrm{KCl}$ on phosphatidylcholine and phosphatidylethanolamine lipid membranes: insight from atomic-scale simulations for understanding salt-induced effects in the plasma membrane. J Phys Chem B 112:1953-1962.

48. Anchordoguy TJ, Carpenter JF, Crowe JH, Crowe LM. 1992. Temperature-dependent perturbation of phospholipid bilayers by dimethylsulfoxide. Biochim Biophys Acta 1104:117-122.

49. Rall WF, Fahy GM. 1985. Ice-free cryopreservation of mouse embryos at $-196^{\circ} \mathrm{C}$ by vitrification. Nature 313:573-575.

50. Ahkong QF, Fisher D, Tampion W, Lucy JA. 1975. Mechanisms of cell fusion. Nature 253:194-195.

51. Notman R, Noro M, O'Malley B, Anwar J. 2006. Molecular basis for dimethylsulfoxide (DMSO) action on lipid membrane. J Am Chem Soc 128:13982-13983.

52. Gurtovenko AA, Anwar J. 2007. Modulating the structure and properties of cell membranes: the molecular mechanism of action of dimethyl sulfoxide. J Phys Chem B 111:10453-10460.

53. Gurtovenko AA, Anwar J. 2007. Ion transport through chemically induced pores in protein-free phospholipid membranes. J Phys Chem B 111:13379-13382.

54. Gurtovenko AA, Onike OI, Anwar J. 2008. Chemically-induced phospholipid translocation across biological membranes. Langmuir 24:9656-9660.

55. Vernier PT, Ziegler MJ, Sun Y, Chang WV, Gundersen MA, Tieleman DP. 2006. Nanopore formation and phosphatidylserine externalization in a phospholipid bilayer at high transmembrane potential. J Am Chem Soc 128:6288-6289.

56. Vernier PT, Ziegler MJ, Sun Y, Gundersen MA, Tieleman DP. 2006. Nano-pore facilitated, voltage-driven phosphatidylserine translocation in lipid bilayer — in cells and in silico. Phys Biol 3:233-247.

57. Rog T, Stimson LM, Pasenkiewicz-Gierula M, Vattulainen I, Karttunen M. 2008. Replacing the cholesterol hydroxyl group by the ketone group facilitates sterol flip-flop and promotes membrane fluidity. $J$ Phys Chem B 112:1946-1952. 
58. Marrink SJ, de Vries AH, Harroun TA, Katsaras J, Wassall SR. 2008. Cholesterol shows preference for the interior of polyunsaturated lipid membranes. J Am Chem Soc 130:10-11.

59. Boon JM, Smith BD. 2002. Chemical control of phospholipid distribution across bilayer membranes. Med Res Rev 22:251-281.

60. Dressler V, Schwister K, Haest CWM, Deuticke B. 1983. Dielectric breakdown of the erythrocyte membrane enhances transbilayer mobility of phospholipids. Biochim Biophys Acta 732:304-307.

61. Falck E, Rog T, Karttunen M, Vattulainen I. 2008. Lateral diffusion in lipid membranes through collective flows. J Am Chem Soc 130:44-45.

62. McIntosh TJ, Simon SA. 2006. Role of bilayer material properties in function and distribution of membrane properties. Annu Rev Biophys Biomol Struct 35:177-198.

63. Lundbaek JA. 2006. Regulation of membrane protein function by lipid bilayer elasticity — a single molecule technology to measure the bilayer properties experienced by an embedded protein. J Phys Cond Matter 18: S1305-S1344.

64. Holthuis JCM, van Meer G, Huitema K. 2003. Lipid microdomains, lipid translocation and the organization of intracellular membrane transport. Mol Membr Biol 20:231-241.

65. Schekman R. 2004. Merging cultures in the study of membrane traffic. Nat Cell Biol 6:483-486.

66. Holthuis JCM, Levine TP. 2005. Lipid traffic: floppy drives and a superhighway. Nat Rev Mol Cell Biol 6:209220.

67. Lenoir G, Williamson P, Holthuis JC. 2007. On the origin of lipid asymmetry: the flip side of ion transport. Curr Opin Chem Biol 11:654-661.

68. Bockmann RA, de Groot BL, Kakorin S, Neumann E, Grubmuller H. 2008. Kinetics, statistics, and energetics of lipid membrane electroporation studied by molecular dynamics simulations. Biophys J 95:1837-1850. 